\title{
Effects of Input Device Familiarity on Content Creation and Sharing in Meetings
}

\author{
Himanshu Verma \\ EPFL \\ 1015 Lausanne \\ Switzerland \\ h.verma@epfl.ch \\ Flaviu Roman \\ EPFL \\ 1015 Lausanne \\ Switzerland \\ flaviu.roman@epfl.ch \\ Patrick Jermann \\ EPFL \\ 1015 Lausanne \\ Switzerland \\ patrick.jermann@epfl.ch \\ Pierre Dillenbourg \\ EPFL \\ 1015 Lausanne \\ Switzerland \\ pierre.dillenbourg@epfl.ch
}

Copyright is held by the author/owner(s)

CHI'12, May 5-10, 2012, Austin, Texas, USA.

ACM 978-1-4503-1016-1/12/05.

\begin{abstract}
In co-located meetings, content creation is often distributed among the group members and sharing requires transfer of content artifacts, which impedes collaboration. In this paper, we present the design of a collaborative environment to support this activity in meetings for small groups. The system consists of a shared wall-mounted workspace where users can interact using either mouse and keyboard or digital pen and paper. We also present a user study comparing the two input configurations and its preliminary results.
\end{abstract}

\section{Author Keywords}

Single display groupware; co-located collaboration; shared workspaces; multiple input devices; CSCW

\section{ACM Classification Keywords}

H.5.3 [Information Interfaces and Presentation]: Group and Organization Interfaces - Computer-supported cooperative work.

General Terms

Human Factors, Experimentation

\section{Introduction}

Meetings are the widely accepted way of decision making and problem solving in a collaborative way. Creation of 


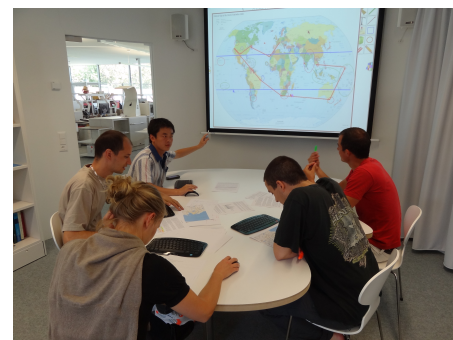

Figure 1: Collaborative environment with Mouse and Keyboard setup.

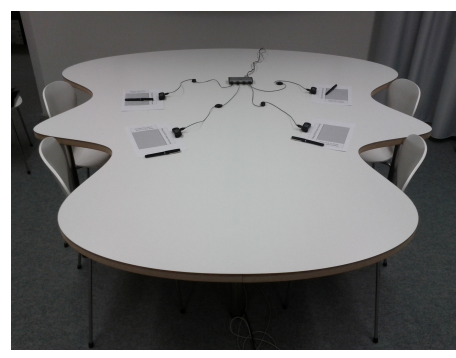

Figure 2: Meeting table and the Pen and Paper setup. artifacts such as written notes, drawings etc. is not the main activity in meetings, but assists participants to capture ideas and opinions which could be relevant to the meeting goals. However content creation is a distributed activity as attendees have their own copy of notes, and they are not aware of their peers' action. In order to support an idea or express their opinion, participants share these artifacts among each other. Sharing requires either the transfer of artifacts to others or explicit acquisition of control over the shared resources such as a flip-chart.

This might lead to reduced visibility of the shared artifact in the group and floor monopolization. We hypothesize that a Single Display Groupware (SDG) as defined by Stewart et al. [9] can mitigate these issues. SDG embodies the benefits of a shared workspace coupled with simultaneous interaction ability, enabled by the multiple input devices - one for each user.

Thus, we propose a collaborative meeting environment with input devices for each participant to interact simultaneously over a shared wall-mounted display.

\section{Related Work}

Many researchers have tried to study SDGs in diverse scenarios ranging from education of school children [7] to organizational meetings $[4,5]$. It is also identified that shared workspaces as comprised in SDG, offer potential benefits like increased awareness, facilitate the process of grounding and could act as a group memory due to the persistence of information shared over it as examined by Dillenbourg [1].

However, we observe that the existing SDG systems are restricted in the way users can interact with the system. For example, the interaction with the shared display is limited to individual claimed spaces in Dynamo [4] and users have to compete for the screen space in order to share. In PointRight [5] the interaction itself is restricted due to technological constraints and the system allows only one pointer per display, thus reducing chances of collaboration. Also, some SDG systems require multi-touch tables to interact with the system as in WeSpace [11]. This can be cumbersome to use, as shared content on the table has reduced visibility among the group and prolonged usage results in fatigue [10]. In addition, these systems require users to learn the functionality of the system and perform extra effort every time to keep it operable, such as connecting individual laptops or PDAs to share content $[4,11]$. Despite the presence of varied systems supporting meetings, paper and pen are still preferred over their digital counterparts due to user's familiarity with them and absolute ease of use. It is thus desired to design novel interfaces which enable users to use paper and pen to interact with digital media during collaboration as presented by Haller et al. [2].

\section{Technical Setup and Tools}

Our proposed system design incorporates a front projected public display of size $2 \mathrm{~m} \times 2 \mathrm{~m}$, as well as a meeting table placed in front of the public display (see Fig. 1). The meeting table has a specific shape (wave like shape as shown in Fig. 2) designed to ergonomically facilitate face-to-face interaction between participants as well as interaction with the public display. The system consists of two setups - in the first setup each user is equipped with a wireless mouse and keyboard (MK), while in the other each user is supplied with a digital pen ${ }^{1}$ and paper (PP). Internally by the system, the input from a pen is handled similar to a mouse. Each input device is color coded, which helps users to identify their respective cursors on the public display in both the setups. Finally, the public display as well as the input devices are connected to a single computer.

\footnotetext{
${ }^{1}$ IRISNotes Pen : www.irisnotes.de
} 


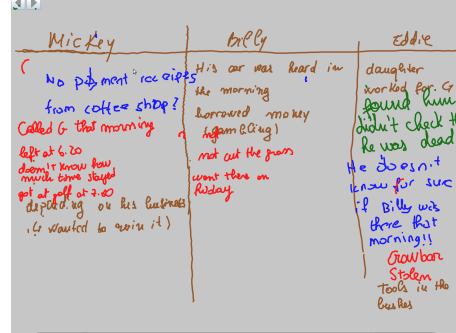

Figure 3: Snapshot of the shared workspace for the pen and paper setup. Each color denotes a different user.
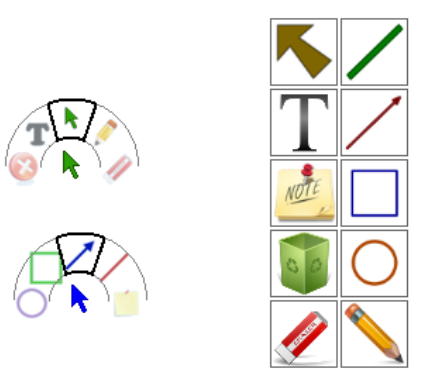

Figure 4: Snapshot of the two tool collections in the mouse and keyboard setup. On the right hand side, there is the fixed tool collection (public to all group members). While on the left there are the scrollable tool collections individual to each mouse cursor.

\section{Design Rationale}

Wallace et al. [10] have already established that, SDG is beneficial for coordinating over the shared resources whereas multi-display groupware (MDG) is advantageous for individual task duties. This was our initial design decision to select SDG over MDG. Next, we target mouse and keyboard as the preferable input devices instead of multi-touch tables. Mice offer a higher degree of pointing accuracy and keyboard is a faster, convenient and robust means of entering text as compared to touch based or on-screen keyboards. Furthermore, a comparison between multi-mouse SDG and multi-touch table suggests that, users are more efficient with the former setup, but prefer the later [3].

The absolute ease of use of pen and paper renders it the preferred tool during collaboration. Also, creation of content such as drawings and schemas is considerably quicker and more efficient using a pen instead of a mouse. This prompts us to use multiple pens during collaboration as the availability of a pen for each user will reduce the chances of a user acquiring explicit control over the shared resource, thus moderating floor monopolization.

\section{Shared Workspace over Public Display}

Similar to the conventional paper-based flip charts, we use a page metaphor for our shared workspace. Unlike conventional flip charts our shared workspace allows multiple users to simultaneously add and manipulate artifacts such as graphics, text and images. In case of space shortage on the current page, users can create a new blank page while concurrently saving the previous works. In the mouse and keyboard setup, the group is provided with a Page Manager widget designed to enable the group to browse through previously created pages.

Whereas in the pen setup, users are provided with normal A4 sheets upon which is marked the area mapped to the entire public display. Anything written within this area is concurrently reflected on the public display in the color of the device color code (see Fig. 3). Changing of page on the public display can be accomplished by tapping the pen over one of the two squares printed on the paper indicating the direction of the page change.

\section{Tool Collection}

We wanted to provide users only with the minimal functionality necessary to support the task at hand, as abundance of tools consumes a lot of screen space and involves higher anticipated learning efforts and time. Also, content creation and sharing is not the main activity during meetings, instead a supporting activity going on in the background. In the mouse and keyboard setup the basic toolset includes lines, arrows, ellipse, rectangle, free-hand drawing and post-its. The users are provided with two toolsets containing the above mentioned functionalities. While one of the toolbars is personal to each mouse pointer, the other one is public for everyone to use (see Fig. 4).

In the pen system no additional tools are provided to the user, like an ability to create predefined shapes from strokes on the paper. The pen is intended to be used in the natural way.

\section{User Study}

In designing a groupware, an important aspect of the evaluation is to understand the role of input device familiarity on group dynamics, and how it influences the task outcome. We hypothesize that presence of a familiar input mechanism will motivate users to share more and thus improve the quality of solution. Therefore, we conducted a user study to investigate the differences between the multi-pen setup and the multi-mouse (and keyboard) setup. 


\section{Participants}

Sixty-six participants (23 females and 43 males) aged 17-35 years (average age of 23) were recruited for the study, from the Swiss Federal Institute of Technology, Lausanne. Groups of four participants conducted the experiment task, except for two cases where the experiment was performed with a group of 3 participants. Participants were paid 25 Swiss Francs each for their participation in the study.

\section{Experimental Task}

During the experiment, each group was asked to complete the murder mystery task designed by Stasser et al. [8], where the group members were required to use the collaborative environment to generate and share content. This is a hidden profile task where some crucial information about the suspects is left unshared among the group. The correct identification of murderer requires the group members to combine the information by sharing and discussing as much as possible, as incomplete information might lead to inferior solutions and premature solution convergence [6]. This task was chosen because it resembles the collaborative problem solving strategy, where the group has some shared knowledge about the task as well as some unshared information which could prove to be important towards the end result. In addition, the choice of the input device to create and share information using a groupware might influence the sharing behavior of participants. Groups using different collaborative tools (mouse and keyboard versus pen and paper) might have different shared representations of the murder mystery.

\section{Design}

We used a two input configurations, between-subjects design for our user study. The two input configurations for our collaborative environment are namely - mouse and keyboard (MK) and pen and paper (PP). Groups of 4 participants completed the experimental task in one of the two conditions. The task was not time bound, but an upper limit of 2 hours was set and the groups were asked to suggest the name of the suspect most likely to have committed the crime. However, the average time required to complete the task by all the groups was 90 minutes. In total, there were 17 groups during the study ( 15 groups with 4 participants, 2 groups with 3 participants), where 9 groups completed the experiment in MK condition and 8 groups in PP condition.

\section{Data Collection}

Each participant was asked to complete two questionnaires; one before and one after the study. The pre-experiment questionnaire collected basic information about participants. The post experiment questionnaire contained statements which the participant had to agree or disagree with. We used a 5-point Likert scale to record their agreement, with 1 as strong disagreement and 5 as strong agreement.

In addition, two observers manually recorded the number of deictic gestures and utterances for each participant in the group. The utterances were classified either to be relating to the task or to the organization of group activities. Similarly, deictic gestures were divided so as to refer to the task material (booklets given to the participants) or to the content shared on the public display. Also, all the interactions with the collaborative environment were recorded in system log files.

\section{Results and Analysis}

One way ANOVA of the experiment data reveals that, groups in the PP condition completed the task in significantly less time as compared to the MK condition 
$(F(1,64)=52.2419, p<0.01)$. This could be explained by the fact that familiarity with the input object enables the faster sharing of information. However, there is no significant difference of success in the task across the two conditions. There is also a marginally significant difference in the amount of text generated using the groupware across the two conditions, with more text generated in the MK condition $(F(1,64)=4.0829, p=0.04)$. More drawing elements were created in the PP condition, but this difference is not significant. Also, significantly more pages were created $(p=0.01)$ and more switches between pages were made $(p<0.01)$ in the PP condition.

Factor Analysis

We separated the experiment variables into two sets before performing the factor analysis. The first set regards the collaboration (or teamwork) among the group members, while the second set considers the groupware usage (or taskwork) during the task. This division was made based on the classification made by Wallace et al. [10].

We observed that group members who considered themselves extrovert, also participated actively during the task and had higher number of utterances, gestures and shared more information. On the contrary, subjects who preferred to work alone had less participation in the group. Further, the individuals whose contributions were accepted by the group tended to participate actively and vice versa. Also, higher level of engagement of the group members lead to the feeling that the group converged to a common consensus. Finally, the difference in opinions of the group members about the murderer lead to higher acceptance of contributions by the group.

The second factor analysis provides some interesting aspects about groupware usage. We observe that, if it is hard to coordinate using a groupware (more coordination breakdowns due to the overlapping of shared information on the public display or group members simultaneously using the same part of the display), the groupware is not used for a conceptual representation of the murder mystery, such as timeline of important events. Instead it is used as a tool to share information which is referred later during discussion. We also found that there were more coordination breakdowns in the PP condition than in the MK condition. However, this difference is not statistically significant.

\section{Conclusion and Future Work}

We have presented a collaborative meeting environment to support content creation and sharing in co-located meetings, which enables group members to concurrently interact with the shared workspace while mitigating issues such as floor monopolization. The collaborative environment is composed of a shared wall-mounted workspace and two different input configurations. We also conducted a user study to compare these two configurations. We observed that input device familiarity increases the efficiency of the task, and the amount of information shared depends on the affordances of the input device in usage. The factor analysis also emphasizes some crucial aspects of collaboration and group dynamics, such as the enthusiasm exhibited by a participant during collaboration generates a sense of trustworthiness among the others.

\section{Future Work}

The data analysis of the experiment is not yet complete, and we have only presented some initial results in this article. Thus we will perform more analysis on the data. We would also like to investigate the influence of simultaneous interaction ability on the regulation of emerging social structure within the group and if this social structure changes over time. Also, it would be 
interesting to study if concurrent interaction with the shared workspace affects the sense of ownership over an individual's artifacts.

\section{Acknowledgements}

We would like to thank the anonymous reviewers for their comments. Thanks to Cristián Alcoholado Moenne,

Olivier Guédat for their contribution to the development of this system. Thanks to all the students who

volunteered to observe the meeting sessions, also special thanks to all the students who participated in the study.

\section{References}

[1] P. Dillenbourg and D. Traum. Does a shared screen make a shared solution? In Proc. CSCL.

International Society of the Learning Sciences, 1999.

[2] M. Haller, J. Leitner, T. Seifried, J. R. Wallace, S. D. Scott, C. Richter, P. Brandl, A. Gokcezade, and S. Hunter. The NiCE Discussion Room: Integrating paper and digital media to support co-located group meetings. In Proc. CHI, pages 609-618. ACM, 2010.

[3] T. E. Hansen and J. P. Hourcade. Comparing multi-touch tabletops and multi-mouse single-display groupware setups. In Proc. MexIHC, pages 36-43, 2010.

[4] S. Izadi, H. Brignull, T. Rodden, Y. Rogers, and M. Underwood. Dynamo: A public interactive surface supporting the cooperative sharing and exchange of media. In Proc. UIST, pages 159-168. ACM, 2003.

[5] B. Johanson, G. Hutchins, T. Winograd, and M. Stone. PointRight: Experience with flexible input redirection in interactive workspaces. In Proc. UIST, pages 227-234. ACM, 2002.
[6] B. E. Mennecke. Using Group Support Systems to discover hidden profiles: An examination of the influence of group size and meeting structures on information sharing and decision quality.

International Journal of Human-Computer Studies, 47(3):387 - 405, 1997.

[7] N. Moraveji, K. Inkpen, E. Cutrell, and R. Balakrishnan. A Mischief of Mice: Examining children's performance in Single Display Groupware systems with 1 to 32 mice. In Proc. CHI, pages 2157-2166. ACM, 2009.

[8] G. Stasser and D. Stewart. Discovery of hidden profiles by Decision-Making groups: Solving a problem versus making a judgment. Journal of Personality and Social Psychology, 63(3):426-434, Sept. 1992.

[9] J. Stewart, B. B. Bederson, and A. Druin. Single display groupware: a model for co-present collaboration. In Proc. CHI, pages 286-293. ACM, 1999.

[10] J. Wallace, S. Scott, T. Stutz, T. Enns, and K. Inkpen. Investigating Teamwork and Taskwork in Single- and Multi-display groupware systems. Personal and Ubiquitous Computing, 13:569-581, 2009.

[11] D. Wigdor, H. Jiang, C. Forlines, M. Borkin, and C. Shen. WeSpace: The design development and deployment of a walk-up and share multi-surface visual collaboration system. In Proc. CHI, pages 1237-1246. ACM, 2009. 